\title{
Oviposition behaviour of Phlebotomus argentipes - A laboratory-based study
}

\author{
Vijay Kumar/ ${ }^{+}$, Aarti Rama, Shreekant Kesari, \\ Gouri Sankar Bhunia, Diwakar Singh Dinesh, Pradeep Das
}

Department of Vector Biology and Control, Rajendra Memorial Research Institute of Medical Sciences, Bihar, India

The breeding habitat of sandflies is a little studied and poorly understood phenomenon. More importantly, oviposition behaviour is a largely neglected aspect of sandfly biology and this knowledge gap further undermines our understanding of the biology of sandflies. Pheromones released by the eggs play an important role in identifying good sites for oviposition by female insects. Several recent studies have examined the oviposition pheromone. The present study provides a preliminary report on the oviposition behaviour of Phlebotomus argentipes, the only vector of kala-azar (or visceral leishmaniasis) on the Indian sub-continent. Sandflies prefer to oviposit their eggs on surfaces that contain organic substances, especially substances with an odour of decaying animal products and the remains of conspecific eggs. The results presented here suggest that the odour released by the organic substances of old sandfly colony remains that contain dead flies, old unhatched eggs, larval food containing vertebrate faeces, frass and other organic matter serves as an attractant for the ovipositing females of $\mathrm{P}$. argentipes and hence greatly increases the number of oviposited eggs compared to eggs deposited in controlled oviposition pots. This result will be helpful in maintaining an efficient colony of $\mathrm{P}$. argentipes and may be a promising tool for monitoring and controlling the target insect as part of a synergistic approach.

Key words: pheromones - frass - oviposition - conspecific eggs - kala-azar - visceral leishmaniasis

Phlebotomus argentipes, the vector of Indian kalaazar, has received much attention because of its transmission of Leishmania donovani (Mukhopadhyay \& Mishra 1991). Surprisingly, very little information is available on the breeding habitats of $P$. argentipes. Consequently, the lack of information on the breeding of sandflies prevents us from locating these habitats and inhibits the targeting of prevention measures against the pre-imaginal stages of sandflies.

To study sandfly biology and behaviour and to search for new methods of vector control, it is necessary to maintain large laboratory colonies of sandflies. In this respect, it is important to note that oviposition behaviour is a neglected aspect of the biology of sandflies. Pheromones influence oviposition behaviour in the females of haematophagous insects. These pheromones are produced by the ovipositing female or by conspecific eggs that co-occur with gravid females. Moreover, there has been no definitive information from laboratory studies of the oviposition of $P$. argentipes (Ghosh \& Bhattacharya 1992, Dinesh et al. 2009, 2011), although it is thought that the insects select the oviposition site based on chemical factors released by the emerging progeny. It has been observed that in the wild, the behavioural

doi: 10.1590/0074-0276130003

+ Corresponding author: vijay_rmri@sily.com

Received 3 January 2013

Accepted 12 April 2013 ecology of $P$. argentipes is similar to that of Lutzomyia longipalpis (the corresponding South American vector) in terms of lek formation on the blood-feeding hosts, to which females are attracted. Dougherty et al. (1993), with the help of a bio-assay, demonstrated the semichemical-mediated oviposition attraction of gravid $L u$. longipalpis. When determining the location of a suitable oviposition site for egg laying (Dougherty et al. 1993) or a potential host for blood feeding (Kumar et al. 2011), haematophagous insects primarily use their olfactory senses. Previous research conducted by Elnaiem and Ward (1992) demonstrated that Lu. longipalpis preferred sites for oviposition that contain frass or rabbit faeces, which act as its larval rearing medium. More recently, Wasserberg and Rowton (2011) demonstrated that in choice and no-choice oviposition chambers, the effect of old sandfly colony remains (frass), conspecific eggs and their combination affected the oviposition rates of sandflies (Peterkova-Koci et al. 2012). The present study will contribute to the development of a methodology for the maintenance of sandfly colonies, which is a major challenge for scientists working on different biological aspects of sandflies. Additionally, this study may provide promising tools for the monitoring and control of target insects using integrated vector management strategies involving the use of traps baited with pheromones, infusions and insecticides. In the present study, experiments were conducted in Hilton's pots that contained old remains of colonies, including eggs, larvae and larval foods, with empty pots serving as the control group. Hence, in the present study, an attempt was made to observe the possible role of pheromone/odour in the oviposition behaviour of $P$. argentipes. 
In the present study, 100 Hilton pots, including 50 fresh pots and 50 previously used, were kept in the insectorium of Rajendra Memorial Research Institute of Medical Sciences (RMRIMS), Patna, Bihar (India). In this research, the fresh pots served as the control and the old pots with organic medium were considered as experimental pots. The 50 new Hilton pots were prepared using the following steps. A plastic can $(7 \times 7$ $\mathrm{cm}$ ) with a lid was used for each pot; five holes were cut into its base and a large hole was cut in the centre of its lid. A solution of plaster of Paris (POP) $\left[\left(\mathrm{CaSO}_{4}\right)_{2}\right.$. $\left.1 / 2 \mathrm{H}_{2} \mathrm{O}\right]$ and water in a 2:3 ratio was prepared. The POP solution was poured onto a plastic sheet and the drilled plastic pot was firmly placed over it such that the POP solution penetrated inside the can through the holes at its base. Subsequently, the remaining POP solution was slowly poured into the pot to fill it a depth of $1 / 2 \mathrm{~cm}$ and the pot was left for approximately 15 min to dry and solidify. After completely hardening, the pots were cleaned and prepared to house adult sandflies for oviposition. These pots were numbered 1-50 and marked as new pots (control pots). The old Hilton pots (numbered 50-100) containing larval rearing medium, i.e., larval food (a mixture of rabbit faeces and sand in an equal ratio) and the dead remains of larvae, exuviae and eggs, were considered as experimental pots. The laboratorybased oviposition behaviour of sandflies was examined. $P$. argentipes sandflies were collected from the insectarium of RMRIMS.

Newly emerged $P$. argentipes, both male and female, were collected from the colony and provided with glucose after their emergence. The next morning, blood meals from a rabbit were provided to the sandflies. Later, individual, fully blood-fed adult females were captured from the colony using the aspiration technique and were carefully inserted in the Hilton pot (i.e., the oviposition jar). Single females of $P$. argentipes along with a single male $P$. argentipes were inserted in each Hilton pot.

A damp cotton cloth was placed at the bottom of each pot to supply humidity $(80 \% \pm 5 \%)$ and the temperature was maintained at $28^{\circ} \mathrm{C} \pm 2^{\circ} \mathrm{C}$. The pots were examined daily for egg laying.

After the sixth day of confinement, the dead sandflies were carefully removed from the experimental pots with a needle to observe and count the eggs. The eggs were counted using a stereoscopic microscope (Carl Zeiss Stereoscopy Microscope, Austria; Model
426126). On the basis of the obtained data, comparisons were made between the number of eggs in the old pots and new pots.

Descriptive statistics of the sandfly oviposition behaviour were calculated using a Microsoft Excel worksheet. A one-way ANOVA was used to measure the significant differences between the two groups.

The comparative analysis showed distinct differences in the oviposition of $P$. argentipes females between old Hilton pots and fresh pots. The number and the percentage of females that oviposited and the mean number of eggs laid by a single sandfly are presented in Table. A total of 2,962 eggs were oviposited in the experimental pots, whereas only 1,436 eggs were oviposited in the fresh pots. The result of our analysis also illustrated that the experimental pots had a significantly greater number of eggs in comparison to the control pots (F-critical 3.94; $\mathrm{p}$-value 5.096E-13). The average number of ovipositions was 59.24 in the experimental pots, while 28.72 ovipositions were recorded in the control pots. The values of skewness and kurtosis in both the control and experimental oviposition pots were greater than \pm 1 .

The study clearly demonstrated a significant difference in the number of eggs laid in the old Hilton pots in comparison to the fresh Hilton pots $(p<0.001)$. One possible explanation for this difference is that the chemical residues in the old Hilton pots (i.e., the experimental pots), which contained frass, larval food and the dead remains of eggs, exuviae and eggs, may act as a stimulant for oviposition by female sandflies. In a previous study, Dia Eldin et al. (1992) reported that the aroma from frass acts strongly as an ovipositor attractant for mature adult female sandflies and a significantly higher number of eggs was laid in test chambers containing rabbit faeces than in an untreated control chamber. Recently, Wasserberg and Rowton (2011) reported that old colony remains have a positive influence on the oviposition of $\mathrm{Lu}$. longipalpis. Our findings corroborate these works.

Future work is suggested to study the comparative role of pheromones and aromas that are released by conspecific eggs, frass and larval food on the oviposition behaviour of $P$. argentipes. This information may aid in $P$. argentipes colonisation and in formulating effective control strategies, as traps baited with pheromones, infusions and insecticides are promising tools for the monitoring and control of target insects using integrated vector management strategies.

TABLE

Productivity of Phlebotomus argentipes in the experimental and control pots of the colony

\begin{tabular}{lccccccccc}
\hline & $\begin{array}{c}\text { Fed } \\
\text { female } \\
\text { Observation }\end{array}$ & $\begin{array}{c}\text { Fly } \\
\text { oviposited } \\
(\mathrm{n})\end{array}$ & $\begin{array}{c}\text { Total eggs } \\
\text { oviposited } \\
(\mathrm{n})\end{array}$ & Average & Median & $\begin{array}{c}\text { Standard } \\
\text { error }\end{array}$ & $\begin{array}{c}\text { Standard } \\
\text { deviation }\end{array}$ & Kurtosis & Skewness \\
\hline Control & 50 & 50 & 1,436 & 28.72 & 26 & 2.43 & 17.17 & 0.36 & 0.87 \\
Experimental & 50 & 50 & 2,962 & 59.24 & 58 & 2.64 & 18.67 & -0.06 & 0.52 \\
\hline
\end{tabular}




\section{ACKNOWLEDGEMENTS}

To Mr NK Sinha, AK Mandal and SA Khan (RMRIMS), for technical support, to Ujjwal Prakash and Lokesh Shankar, for assistance to carry out the research work, and to RMRI Institutional Ethical Committee and the Animal Ethics Committee of Indian Council of Medical Research, Government of India, for giving mandatory approval to the present research work involving experiments using animals and rearing sandfly cultures in RMRIMS insectorium.

\section{REFERENCES}

Dia-Eldin, Elnaiem A, Ward R 1992. Oviposition attractants and stimulants for the sandfly Lutzomyia longipalpis (Diptera: Psychodidae). J Med Entomol 29: 5-12.

Dinesh DS, Kumar AJ, Kumar V, Ranjan A, Das P 2011. Fecundity and life table of different morphotypes of Phlebotomus argentipes (Diptera: Psychodidae). J Parasit Dis 35: 113-115.

Dinesh DS, Singh A, Kumar V, Kesari S, Kumar AJ, Kishore K, Roy SP, Bhattacharya SK, Das P 2009. Emergence periodicity of Phlebotomus argentipes Annandale and Brunetti (Diptera: Psychodidae): a laboratory study. J Parasit Dis 33: 23-27.
Dougherty MJ, Hamilton JGC, Ward RD 1993. Semiochemical mediation of oviposition by the phlebotomine sand fly Lutzomiya longipalpis. Med Vet Entomol 7: 219-224.

Elnaiem DA, Ward RD 1992. The thigmotropic oviposition response of the sandfly Lutzomyia longipalpis (Diptera: Psychodidae) to crevices. Ann Trop Med Parasitol 86: 425-430.

Ghosh KN, Bhattacharya A 1992. Gonotrophic nature of Phlebotomus argentipes (Diptera: Psychodidae) in the laboratory. Rev Inst Med Trop Sao Paulo 34: 181-182.

Kumar V, Kesari S, Kumari K, Krishnakumari B, Venugopalan R, Das $P$ 2011. Comparison of in vivo host animals as blood-feeding source for laboratory rearing of the sandfly vector Phlebotomus argentipes (Diptera: Psychodidae). Ann Entomol Soc Am 104: 429-433.

Mukhopadhyay AK, Mishra RN 1991. Development of Leishmania donovani in Phlebotomus argentipes \& Ph. papatasi fed on kalaazar patients in Bihar. Indian J Med Res 93: 152-154.

Peterkova-Koci K, Robles-Murguia M, Ramalho-Ortigão M, Zurek L 2012. Significance of bacteria in oviposition and larval development of the sand fly Lutzomyia longipalpis. Parasit Vectors 5: 145.

Wasserberg G, Rowton ED 2011. Sub-additive effect of conspecific eggs and frass on oviposition rate of Lutzomyia longipalpis and Phlebotomus papatasi. J Vector Ecol 36 (Suppl. 1): S138-S143. 